\title{
A Curiosity of Low-Order Explicit Runge-Kutta Methods
}

\author{
By J. Oliver
}

\begin{abstract}
By introducing an additional parameter into the first stage of the explicit Runge-Kutta process, new formulae of second and third order are derived, offering improved error bounds in the second-order case.
\end{abstract}

1. Introduction. The first stage in an explicit Runge-Kutta process for the solution of an initial-value problem for a system of ordinary differential equations, $y^{\prime}=$ $f(x, y)$, at any point $\left(x_{n}, y_{n}\right)$, has hitherto been presented as being necessarily the evaluation of $k_{1}=h f\left(x_{n}, y_{n}\right)$. While there is no alternative to using the current known value $y_{n}$ if the method is indeed to be explicit in the sense of Butcher [4], there is no corresponding computational reason why $x_{n}$ should not be replaced by $x_{n}+\alpha_{1} h$, for some $\alpha_{1}$ chosen in the same way as the other parameters in the Runge-Kutta process. It might seem at first sight that this additional parameter would introduce an extra degree of freedom into the algebraic equations governing the parameters, and we shall show that this is indeed the case for the two-stage process. Furthermore, we obtain a new two-stage second-order method for which the truncation error bound is smaller than the previous minimum error bound found by Ralston [1]. In the three-stage case, we find that the number of degrees of freedom is in fact reduced by choosing $\alpha_{1}$ to be nonzero, but nevertheless one such family of methods exists, while for four stages the parameters are over-determined unless $\alpha_{1}=0$.

The fact that $\alpha_{1}$ must necessarily vanish for fourth- and higher-order methods is one possible explanation of why earlier authors have apparently overlooked this exception to the general rule in the second-and third-order cases. Another reason may be the practice [3], [4] of considering the differential system $y^{\prime}=f(y)$ in which $x$ is treated as a dependent variable whose derivative is unity. Although it is usually true, as Butcher [3] claims, that no loss of generality results from taking $f$ to be independent of $x$, what follows here can be viewed as a counterexample to this assertion.

2. The General Equations. Following Ralston [1], [2] we write the general explicit Runge-Kutta method with $m$ stages as

$$
y_{n+1}-y_{n}=\sum_{i=1}^{m} w_{i} k_{i}
$$

where the $w_{i}$ 's are constants and

$$
k_{i}=h f\left(x_{n}+\alpha_{i} h, y_{n}+\sum_{j=1}^{i-1} \beta_{i j} k_{j}\right)
$$

Received August 9, 1974.

AMS (MOS) subject classifications (1970). Primary 65 L05.

Key words and phrases. Runge-Kutta methods. 
with $h=x_{n+1}-x_{n}$ and the $\alpha_{i}$ 's and $\beta_{i j}$ 's also constants. Defining the operators

$$
D=\partial / \partial x+(\partial / \partial y) f_{n} \text { and } D_{i}=\alpha_{i}(\partial / \partial x)+\left(\sum_{j=1}^{i-1} \beta_{i j}\right)(\partial / \partial y) f_{n},
$$

where $f_{n}=f\left(x_{n}, y_{n}\right)$, and matching both the powers of $h$ up to $h^{4}$ and the operators in the expansion of the right-hand side of $(2.1)$ about $\left(x_{n}, y_{n}\right)$ with the corresponding terms in the Taylor series expansion of $y_{n+1}-y_{n}$, we obtain eight equations in the case $m=4$ (from which the cases $m=2,3$ can readily be obtained). We give these equations in full, since in earlier presentations such as [2] the terms involving $D_{1}$ were absent.

$$
\begin{aligned}
& h:\left(w_{1}+w_{2}+w_{3}+w_{4}\right) f=f, \\
& h^{2}:\left(w_{1} D_{1} f+w_{2} D_{2} f+w_{3} D_{3} f+w_{4} D_{4} f\right)=\frac{1}{2} D f, \\
& h^{3}: \frac{1}{2}\left(w_{1} D_{1}^{2} f+w_{2} D_{2}^{2} f+w_{3} D_{3}^{2} f+w_{4} D_{4}^{2} f\right)=\frac{1}{6} D^{2} f, \\
& f_{y}\left[\left(w_{2} \beta_{21}+w_{3} \beta_{31}+w_{4} \beta_{41}\right) D_{1} f\right. \\
& \left.\quad+\left(w_{3} \beta_{32}+w_{4} \beta_{42}\right) D_{2} f+w_{4} \beta_{43} D_{3} f\right]=\frac{1}{6} f_{y} D f, \\
& h^{4}:\left(w_{1} D_{1}^{3} f+w_{2} D_{2}^{3} f+w_{3} D_{3}^{3} f+w_{4} D_{4}^{3} f\right)=\frac{1}{24} D^{3} f, \\
& \frac{1}{2} f_{y}\left[\left(w_{2} \beta_{21}+w_{3} \beta_{31}+w_{4} \beta_{41}\right) D_{1}^{2} f\right. \\
& \left.\quad+\left(w_{3} \beta_{32}+w_{4} \beta_{42}\right) D_{2}^{2} f+w_{4} \beta_{43} D_{3}^{2} f\right]=\frac{1}{24} f_{y} D^{2} f, \\
& \quad\left[w_{2} D_{2} f_{y} \beta_{21} D_{1} f+w_{3} D_{3} f_{y}\left(\beta_{31} D_{1} f+\beta_{32} D_{2} f\right)\right. \\
& \left.\quad+w_{4} D_{4} f_{y}\left(\beta_{41} D_{1} f+\beta_{42} D_{2} f+\beta_{43} D_{3} f\right)\right]=\frac{1}{8} D f_{y} D f, \\
& f_{y}^{2}\left[\left(w_{3} \beta_{32} \beta_{21}+w_{4} \beta_{42} \beta_{21}+w_{4} \beta_{43} \beta_{31}\right) D_{1} f+w_{4} \beta_{43} \beta_{32} D_{2} f\right]=\frac{1}{24} f_{y}^{2} D f .
\end{aligned}
$$

It is then customary to argue that these equations can only be satisfied independently of $f(x, y)$ if the ratios

$$
D_{j} f / D f \quad(j=1,2,3,4) \quad \text { and } \quad D_{j} f_{y} / D f_{y} \quad(j=2,3,4)
$$

are constant, which implies that

$$
\alpha_{i}=\sum_{j=1}^{i-1} \beta_{i j} \quad(i=1,2,3,4)
$$

and in particular that $\alpha_{1}=0$. We now show, however, that this argument is invalid in the second-order case.

3. Two-Stage Methods. Matching powers of $h$ up to $h^{2}$, and putting $w_{3}=w_{4}=0$ in (2.4) yields the following three equations corresponding to the terms indicated.

$$
\begin{array}{cl}
h f: & w_{1}+w_{2}=1, \\
h^{2} f_{x}: & \alpha_{1} w_{1}+\alpha_{2} w_{2}=1 / 2, \\
h^{2} f_{y} f: & w_{2} \beta_{21}=1 / 2 .
\end{array}
$$


We thus have a two-parameter family when $\alpha_{1} \neq 0$,

$$
\alpha_{2}=\frac{1}{2 w_{2}}+\alpha_{1}\left(1-\frac{1}{w_{2}}\right), \quad \beta_{21}=\frac{1}{2 w_{2}}, \quad w_{1}=1-w_{2},
$$

including as a special case the usual one-parameter family obtained when $\alpha_{1}=0$.

Writing the truncation error $T_{m}$ in an $m$ th-order method, applied to a single differential equation for ease of analysis, as

$$
T_{m}=c_{m} h^{m+1}+O\left(h^{m+2}\right),
$$

the coefficient $c_{2}$ of $h^{3}$ is here given by

$$
c_{2}=\frac{1}{6} D^{2} f-\frac{1}{2}\left(w_{1} D_{1}^{2} f+w_{2} D_{2}^{2} f\right)+\frac{1}{6} f_{y} D f-w_{2} \beta_{21} f_{y} D_{1} f
$$

and, as expected, no choice of the free parameters can make this vanish independently of $f(x, y)$.

Ralston [1], [2] obtained an upper bound on $\left|c_{2}\right|$ by assuming that in a suitable region about $\left(x_{n}, y_{n}\right)$,

$$
|f(x, y)|<M \text { and }\left|\partial^{i+j} f / \partial x^{i} \partial y^{j}\right|<L^{i+j} / M^{j-1},
$$

where $M$ and $L$ are constants and $i+j \leqslant 2$, and he showed that for $\alpha_{1}=0$ the minimum value of this bound was $M L^{2} / 3$, obtained by setting $\alpha_{2}=2 / 3$, and thus

$$
\begin{aligned}
k_{1} & =h f\left(x_{n}, y_{n}\right), \\
k_{2} & =h f\left(x_{n}+2 h / 3, y_{n}+2 k_{1} / 3\right), \\
y_{n+1} & =y_{n}+k_{1} / 4+3 k_{2} / 4 .
\end{aligned}
$$

A discussion of the relevance of this type of bound to practical computation was also given by Ralston [1], [2].

Under this same assumption (3.5), we obtain from (3.4) using (2.3) and (3.2) a corresponding bound when $\alpha_{1} \neq 0$ of

$$
\begin{aligned}
\left|c_{2}\right|<\left[\left|\frac{1}{2}\left(\frac{1}{3}-\alpha_{1}+\alpha_{1}^{2}\right)-\frac{\left(1 / 2-\alpha_{1}\right)^{2}}{2 w_{2}}\right|\right. & +\left|\frac{1}{3}-\frac{1}{2} \alpha_{1}-\frac{\left(1 / 2-\alpha_{1}\right)}{2 w_{2}}\right| \\
& \left.+\frac{1}{2}\left|\frac{1}{3}-\frac{1}{4 w_{2}}\right|+\frac{1}{2}\left|\frac{1}{3}-\alpha_{1}\right|+\frac{1}{6}\right] M L^{2}
\end{aligned}
$$

and investigation shows that this attains a smaller minimum value, namely $7 M L^{2} / 27$, for the method

$$
\begin{aligned}
k_{1} & =h f\left(x_{n}+h / 3, y_{n}\right), \\
k_{2} & =h f\left(x_{n}+5 h / 9, y_{n}+2 k_{1} / 3\right), \\
y_{n+1} & =y_{n}+k_{1} / 4+3 k_{2} / 4 .
\end{aligned}
$$

On the basis of Ralston's truncation error criterion, therefore, (3.8) is preferable to all previously published second-order methods.

4. Three-Stage Methods. When $m=3$, matching of the powers of $h$ up to $h^{3}$ requires that, from (2.4), 


$$
\begin{aligned}
w_{1}+w_{2}+w_{3} & =1, \\
w_{1} D_{1} f+w_{2} D_{2} f+w_{3} D_{3} f & =\frac{1}{2} D f \\
w_{1} D_{1}^{2} f+w_{2} D_{2}^{2} f+w_{3} D_{3}^{2} f & =\frac{1}{3} D^{2} f, \\
\left(w_{2} \beta_{21}+w_{3} \beta_{31}\right) f_{y} D_{1} f+w_{3} \beta_{32} f_{y} D_{2} f & =\frac{1}{6} f_{y} D f .
\end{aligned}
$$

In order to satisfy these equations independently of $f$, we must have

$$
\beta_{21}=\alpha_{2} \text { and } \beta_{31}+\beta_{32}=\alpha_{3}
$$

so that $D_{j} f=\alpha_{j} D f(j=2,3)$. If $\alpha_{1}$ is not to vanish, then since $D_{1} f / D f$ will depend upon $f$, Eqs. (4.1) can only be satisfied by requiring $w_{1}=0$, in which case they reduce to

$$
\begin{gathered}
w_{2}+w_{3}=1, \quad \alpha_{2} w_{2}+\alpha_{3} w_{3}=\frac{1}{2}, \quad \alpha_{2}^{2} w_{2}+\alpha_{3}^{2} w_{3}=\frac{1}{3}, \\
\alpha_{1}\left(w_{2} \alpha_{2}+w_{3} \beta_{31}\right)=0, \quad w_{3} \beta_{32} \alpha_{2}=\frac{1}{6} .
\end{gathered}
$$

Note that the usual choice of $\alpha_{1}=0$ effectively removes the penultimate equation of (4.3), and since $w_{1}$ can then also be nonzero, the system obtained from (4.1) has two degrees of freedom. By contrast, we have here seven equations (4.2) and (4.3) in eight parameters, possessing the one-parameter solution

$$
\alpha_{2}=\beta_{21}=\frac{1}{3}, \quad \alpha_{3}=1, \quad \beta_{31}=-1, \quad \beta_{32}=2, \quad w_{2}=\frac{3}{4}, \quad w_{3}=\frac{1}{4}
$$

with $\alpha_{1}$ arbitrary, and thus specifying the third-order method

$$
\begin{aligned}
& k_{1}=h f\left(x_{n}+\alpha_{1} h ; y_{n}\right), \quad k_{2}=h f\left(x_{n}+h / 3, y_{n}+k_{1} / 3\right), \\
& k_{3}=h f\left(x_{n}+h, y_{n}-k_{1}+2 k_{2}\right), \quad y_{n+1}=y_{n}+3 k_{2} / 4+k_{3} / 4 .
\end{aligned}
$$

The coefficient $c_{3}$ of $h^{4}$ in the truncation error when (4.5) is applied to a single differential equation is

$$
\begin{aligned}
c_{3}= & -\frac{1}{216} D^{3} f+\frac{1}{72} f_{y} D^{2} f-\frac{1}{24}\left[\left(1-4 \alpha_{1}\right) f_{x}+f f_{y}\right] D f_{y} \\
& +\frac{1}{24}\left[\left(1-4 \alpha_{1}\right) f_{x}+f f_{y}\right] f_{y}^{2}
\end{aligned}
$$

and under similar assumptions (3.5) on $f$ and its derivatives for $i+j \leqslant 3$ the resulting bound on $\left|c_{3}\right|$ attains its minimum value of $(47 / 216) M L^{3}$ when $\alpha_{1}=1 / 4$. Unfortunately, this bound is nearly double the value of $M L^{3} / 9$ pertaining to the optimum thirdorder method with $\alpha_{1}=0$ advocated by Ralston [1].

5. Four-Stage Methods. In an attempt to find parameters which satisfy all the Eqs. (2.4) with $\alpha_{1}=0$, thus giving a four-stage method of fourth order, we follow, for the same reasons, the approach which proved successful in the three-stage case and put

$$
\beta_{21}=\alpha_{2}, \quad \beta_{31}+\beta_{32}=\alpha_{3}, \quad \beta_{41}+\beta_{42}+\beta_{43}=\alpha_{4}, \quad w_{1}=0 .
$$


Using (5.1) to substitute for $\beta_{21}, \beta_{31}, \beta_{41}$ and $w_{1}$ in (2.4) and rearranging, we find that the following eleven equations must be satisfied by the ten parameters involved.

$$
\begin{gathered}
w_{2}+w_{3}+w_{4}=1, \quad \alpha_{2} w_{2}+\alpha_{3} w_{3}+\alpha_{4} w_{4}=\frac{1}{2}, \quad \alpha_{2}^{2} w_{2}+\alpha_{3}^{2} w_{3}+\alpha_{4}^{2} w_{4}=\frac{1}{3} \\
\alpha_{2}^{3} w_{2}+\alpha_{3}^{3} w_{3}+\alpha_{4}^{3} w_{4}=\frac{1}{4}, \quad \alpha_{1}\left(w_{3} \beta_{32}+w_{4} \beta_{42}+w_{4} \beta_{43}-\frac{1}{2}\right)=0 \\
\alpha_{2} w_{3} \beta_{32}+\alpha_{2} w_{4} \beta_{42}+\alpha_{3} w_{4} \beta_{43}=\frac{1}{6}, \quad \alpha_{2}^{2} w_{3} \beta_{32}+\alpha_{2}^{2} w_{4} \beta_{42}+\alpha_{3}^{2} w_{4} \beta_{43}=\frac{1}{12}, \\
\alpha_{1}\left(\alpha_{3} w_{3} \beta_{32}+\alpha_{4} w_{4} \beta_{42}+\alpha_{4} w_{4} \beta_{43}-\frac{1}{3}\right)=0 \\
\alpha_{2} \alpha_{3} w_{3} \beta_{32}+\alpha_{2} \alpha_{4} w_{4} \beta_{42}+\alpha_{3} \alpha_{4} w_{4} \beta_{43}=\frac{1}{8} \\
\alpha_{1}\left(w_{4} \beta_{43} \beta_{32}-\frac{1}{6}\right)=0, \quad \alpha_{2} w_{4} \beta_{43} \beta_{32}=\frac{1}{24}
\end{gathered}
$$

It is clear that the usual choice of $\alpha_{1}=0$ effectively removes three of these constraints, leaving only eight equations in nine parameters, and noting that $w_{1}$ need not then be set to zero, there are two degrees of freedom. On the other hand if we insist that $\alpha_{1} \neq 0$, then it is possible though tedious to verify that Eqs. (5.2) are indeed independent, and so no four-stage explicit method with $\alpha_{1} \neq 0$ can be of fourth order. A similar situation applies if more stages are introduced in order to achieve corresponding. ly higher order.

6. Conclusions. We have shown that if the parameter $\alpha_{1}$ is not arbitrarily set to zero, then new families of second and third (but not fourth) order are obtained, and that on the basis of Ralston's truncation error criterion a particular one of these new methods (3.8) should be employed when a second-order Runge-Kutta method is to be used for starting the solution of an initial-value problem or changing the interval.

Computing Centre

University of Essex

Colchester, England

1. A. RALSTON, "Runge-Kutta methods with mimimum error bounds," Math. Comp., v. 16, 1962, pp. 431-437. MR 27 \#940. $32 \# 8479$.

2. A. RALSTON, A First Course in Numerical Analysis, McGraw-Hill, New York, 1965. MR

3. J. C. BUTCHER, "Coefficients for the study of Runge-Kutta integration processes," $J$. Austral. Math. Soc., v. 3, 1963, pp. 185-201. MR 27 \#2109.

4. J. C. BUTCHER, "Implicit Runge-Kutta processes," Math. Comp., v. 18, 1964, pp. 5064. MR $28 \# 2641$. 